\title{
The law of accelerating returns
}

The American technologist Ray Kurzweil is also known as a 'futurist', and he has a certain talent for predicting technological trends. Twenty years ago, for example, Kurzweil predicted that, sometime in the early 21 st century, hand-held devices would enable blind people to read. Sure enough, devices that accurately read printed text aloud have come onto the market. Kurzweil's ability to predict technological advances, he suggests, comes from an appreciation of the 'law of accelerating returns' - the tendency for advances to feed on themselves, increasing the rate of further advance, and pushing well past what one might sensibly project by linear extrapolation of current progress.

By way of illustration, after the project to sequence the human genome took years just to finish $1 \%$ of the job, it seemed that completion would take decades. Instead, methods improved, spurred in part by competition, and the task was completed in only seven years. Advances in methods for gene sequencing may well, in time, illustrate Kurzweil's point even more dramatically, with new physics having a vital role. Our ability to sequence genes will soon be incomparably faster than it is today, and the techniques may be as novel and as important as what they make possible.

Current sequencing methods still rely on chopping DNA into small pieces, and using techniques based on electrophoresis and optical detection to sequence those pieces. It then takes sophisticated postprocessing of the data to stitch the many sub-sequences back together. A fundamental barrier to faster sequencing is the maximum 'read-length' limit in electrophoresis, that is, the longest strand of DNA that can be sequenced in one step. This is now of the order of 1,000 bases, and seems unlikely to get much longer.

There may be a much better way. Imagine that one could grab hold of a DNA strand, without breaking it, and drag it through some device, reading off the sequence of bases along the way. This sounds like science fiction today, yet as Michael Zwolak and Massimiliano Di Ventra describe (Rev. Mod. Phys. 80, 141-165; 2008), numerous experiments are already tantalizingly close to doing something quite similar. What's making it possible is delicate control of polymer dynamics, coupled with elegant techniques for engineering structures on the nanoscale.
Roughly a decade ago, biophysicist John Kasianowicz and collaborators discovered that an applied voltage would, under the correct conditions, pull singlestranded polynucleotides, which have a negatively charged backbone, through a biological nanopore. These initial experiments monitored the polymer movement by measuring temporary reductions in the ionic current normally flowing through the pore from one electrode to the other. In the experiments, they found there was a reduction in current over a time period that increased linearly with the length of the polymer, and fell with increasing voltage, indicating that

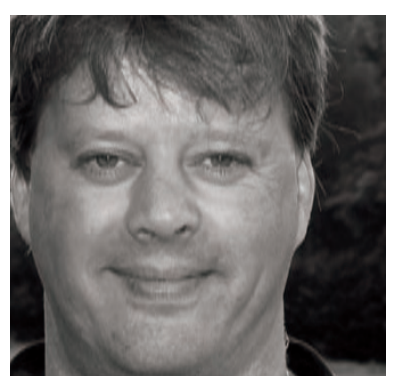

\section{A seemingly trivial capability can raise a host of further possibilities.}

the polymer was blocking the ions while in transit. Further work established that the duration and magnitude of this ionic blockade is dependent on the details of the DNA sequence.

Of course, dragging a polymer through a tiny hole isn't the same as sequencing it. But this seemingly trivial capability raises a host of further possibilities.

One of the most direct possibilities for turning polymer translocation into DNA sequencing might rely on measuring fluctuations in the ionic current caused by the varying properties of each passing base. Suppose a tiny pore were put into a very thin membrane, the pore having a length of only one nucleotide (of the order of $1 \mathrm{~nm}$ ) and a diameter roughly the same. In this case, the volume of the pore would be comparable to that of each base, and these would, when present, block the pore appreciably, triggering changes in the ionic current. Based on nucleotide properties, estimates suggest that structural differences in the bases could lead to current differing by a few percent.
It seems likely, however, that noise either in the ionic current, or caused by structural fluctuations of the nucleotides, would swamp such a signal. Yet creative engineering of pores might easily amplify the effects. Some researchers, by adding appropriate molecules to a pore, have already engineered a system that stirs up ionic currents adequate for differentiating bases with about $95 \%$ accuracy.

Of course, there's also more to work with than the ionic current. Zwolak and Di Ventra suggest a very different route to sequencing that would embed electrodes within a nanopore to induce measurable transverse currents through a passing DNA strand. Although the various DNA bases are indeed quite similar, the differences in their structures are enough to create significant differences in their response when placed between electrodes. If the bases remain well aligned with the electrodes, calculations suggest that the current would differ by at least a factor of two for the nucleotides $\mathrm{G}$ and $\mathrm{C}$, and by orders of magnitude for others.

This would seem to be an exceptionally promising technique, although wrinkles need to be ironed out. For example, there's nothing to guarantee, and much reason to doubt, a beneficial alignment of the bases with the electrodes, and molecular dynamics simulations show that fluctuations in their orientations would themselves cause orders-of-magnitude changes in the current. However, efforts to devise ways of controlling the orientation show promise, especially through the application of transverse electric fields.

These developments have only been underway for a decade, so it seems almost certain that researchers will find other novel ways to get a signal from each passing base. If these technical problems can be overcome, Zwolak and Di Ventra suggest, then a single run through the 3 billion bases on a single strand of human DNA might be done in roughly 7 hours or so - considerably faster than the several months currently required.

If achieved over, say, the next decade, this would be impressive enough progress. But this is the kind of progress we can already see clearly and plan for, and I wonder if we won't be surprised again by Kurzweil's 'law of accelerating returns'. By 2020, Kurzweil might well predict, an individual's genome may be sequenced in under a minute.

Mark Buchanan 